\title{
CtBP2 promotes proliferation and reduces drug sensitivity in non-small cell lung cancer via the Wnt/ $\beta$-catenin pathway
}

\author{
D. P. WANG ${ }^{1, *}$, L. L. GU ${ }^{1, *}$, Q. XUE ${ }^{2}$, H. $\mathrm{CHEN}^{1}$, G. X. MAO ${ }^{1, *}$ \\ ${ }^{1}$ Department of Oncology, Affiliated Hospital of Nantong University, Nantong 226001, Jiangsu, China; ${ }^{2}$ Department of Thoracic Surgery, Affili- \\ ated Hospital of Nantong University, Nantong 226001, Jiangsu, China \\ ${ }^{*}$ Correspondence: maoguoxin33@163.com \\ ${ }^{*}$ Contributed equally to this work.
}

Received May 21, 2017 / Accepted July 22, 2017

\begin{abstract}
The C-terminal binding protein 2 (CtBP2) is crucial for the activation of the Wnt/ $\beta$-catenin pathway and regulates significant cellular processes in multiple cancer cells. However, the role of CtBP2 in non-small cell lung cancer (NSCLC) remains uncertain. Our western blotting and immunohistochemistry assays revealed that CtBP2 expression was obviously increased in NSCLC tissues and cells. In addition, the chi-square test and Kaplan-Meier analysis showed that over-expression of CtBP2 correlates with more invasive tumor phenotype and poor prognosis. In vitro studies with serum starvation-refeeding and CtBP2-shRNA transfection assay demonstrated that CtBP2 expression facilitates NSCLC cell proliferation and reduces sensitivity to cis-diamminedichloroplatinum (CDDP). The possible signaling transduction pathways were investigated, and the immunoprecipitation assay revealed that CtBP2 interacts directly with DvL1. Depletion of CtBP2 resulted in inhibited DvL1 expression and decreased expression of downstream genes. Moreover, our study showed that CtBP2 knockdown enhanced NSCLC cell sensitivity to CDDP through inhibition of the Wnt/ $\beta$-catenin pathway. These results suggest that CtBP2 plays a crucial role in NSCLC progression and CDDP sensitivity, and that CtBP2 depletion can provide a new target for NSCLC treatment.
\end{abstract}

Key words: CtBP2, NSCLC, Wnt/ $\beta$-catenin pathway, proliferation, drug sensitivity

Lung cancer is the most prevalent malignancy in the world and is the leading cause of cancer death [1]. Non-small cell lung cancers (NSCLC), mainly adenocarcinomas and squamous cell carcinomas, account for approximately $80 \%$ of all lung cancers [2]. Despite recent developments in treatment strategies for NSCLC, the prognosis remains unsatisfactory with overall 5-year survival rate of only $10-15 \%$ [3]. Tumorigenesis of lung cancer is characterized by abnormal cell cycle progression associated with aberrant alterations in genes related to cell proliferation regulators. The identification of genes and their mechanisms involved in cell growth modulation is therefore essential to develop effective strategies for diagnosis and therapy in this cancer.

The C-terminal binding protein ( $\mathrm{CtBP}$ ) associated with cellular processes was discovered through its interaction with the C-terminus of the adenovirus E1A protein [4]. The CtBP family is encoded in mammals by two genes: CtBP1 and CtBP2; and CtBP2 is the more important molecule because it is involved in vital cellular processes including cell proliferation and apoptosis $[5,6]$. Evidence shows that
CtBP2 induces the epithelial-to-mesenchymal transition (EMT), mediates repression of tumor suppressor genes, and functions against apoptosis [7]. While CtBP2 regulates Wnt signaling by binding to $\beta$-catenin [8], it is over-expressed in esophageal squamous cell and hepatocellular carcinomas and also in gastric, prostate and ovarian cancers [9-13]. Furthermore, recent studies have found that knockdown of CtBP2 expression suppresses cell proliferation and tumorigenesis in breast cancer-derived cell lines and gastric cancer $[6,9]$. These results show that CtBP2 has an eminent role in both tumorigenesis and tumor progression. However, there has been no complete study into CtBP2 expression in NSCLC, and it is therefore necessary to explore this role and CtBP2's potential mechanisms in NSCLC.

Many studies have revealed that canonical Wnt/ $\beta$-catenin signaling is a classic pathway with crucial roles in cancer progression, including cell proliferation, survival, migration and renewal [14]. The three DvL1, 2 and 3 disheveled proteins have been identified in humans [15], and these are highly conserved components of canonical and noncanonical Wnt 
signaling pathways. Numerous studies have reported that DvLs are over-expressed in various tumor types, including breast cancer, prostate cancer, cervical squamous cell carcinoma, and lung cancer [16]. It has been further confirmed that both DvL-1 mRNA and protein are up-regulated in NSCLC and indicate poor prognosis. DvL-1 affects the biological behavior of NSCLC cells mainly through $\beta$-catenin (the canonical Wnt pathway) [17]. Therefore, CtBP2 may regulate the initiation and cell proliferation of NSCLC via Wnt/ $\beta$-catenin pathway by regulating DvL1.

Our study investigated CtBP2's role in NSCLC progression. CtBP2 expression in 8 paired tumor and adjacent non-tumor tissues were detected by western blot analysis. Immunohistochemistry (IHC) assay was performed in 150 NSCLC samples, and we then examined the correlation between CtBP2 expression and clinical and pathological factors, and assessed its prognostic implications. We then explored the role of CtBP2 expression in regulating cell proliferation and drug sensitivity in NSCLC cells, and our combined results should improve understanding of the mechanisms underlying non-small cell lung cancer development.

\section{Patients and methods}

Patients and tissue samples. Eight paired fresh tumorous and adjacent non-tumorous NSCLC tissues were frozen at $-80^{\circ} \mathrm{C}$ immediately after surgical removal for future use in western blot analysis. For immunohistochemical analysis, all 150 lung cancer sections were from patients who underwent lung resection without pre-operative systemic chemotherapy between 2005 and 2009 at the Surgery Department of the Affiliated Hospital of Nantong University. Prior consent of the patients and approval from the Institutional Research Ethics Committee were obtained for the use of this clinical material. The main clinical and pathologic variables are shown in Tables 1, 2 and 3.

Cell lines and cell culture. The human NSCLC cell lines A549, H1299 and SPCA-1 were acquired from the China Academy of Science Cell Library (Beijing, China). All cells were cultured in RPMI-1640 medium (Gibco-BRL, Grand Island, NY, USA) supplemented with $10 \%$ fetal bovine serum (FBS) in $5 \% \mathrm{CO}_{2}$ at $37^{\circ} \mathrm{C}$.

Western blot analysis. Protein extraction and western blot analysis were performed as previously described [18]. The band intensity was measured by an ImageJ analysis system. The primary antibodies were: anti-DvL1, anti- $\beta$ catenin, anti-PCNA, anti-Cyclin D1, anti-Cyclin A2 and antiGAPDH, and these were obtained from Santa Cruz Biotechnology (Santa Cruz, CA), anti-cleaved PARP (Cell Signaling Technology Shanghai, China), anti-CtBP2 (BD Transduction Laboratories, USA).

Immunohistochemistry. The sections were de-paraffinised using a graded ethanol series, and endogenous peroxidase activity was blocked by soaking in $0.3 \%$ hydrogen peroxide for $20 \mathrm{~min}$. Sections were then processed in
$10 \mathrm{mmol} / \mathrm{L}$ citrate buffer ( $\mathrm{pH} 6.0$ ) and heated to $121^{\circ} \mathrm{C}$ in an autoclave for $3 \mathrm{~min}$ to retrieve the antigen. After rinsing in PBS (pH7.2), the sections were subsequently incubated with anti-CtBP2 antibody (diluted 1:100; BD) for overnight at $4{ }^{\circ} \mathrm{C}$ and anti-Ki-67 antibody (diluted 1:100; Millipore) for $2 \mathrm{~h}$ at room temperature. All slides were processed using the peroxidase-anti-peroxidase method (DAKO, Hamburg, Germany). After rinsing in PBS, the peroxidase reaction was visualized by incubating the sections with DAB. After rinsing with water, the sections were counterstained with hematoxylin, dehydrated and cover slipped.

Immunohistochemical evaluation. All immunostained sections were evaluated in a blind-manner without knowledge of the patients' clinical and pathological parameters. For assessment of CtBP2 and Ki-67, five views were chosen per slide, and at least 1000 cells were counted per view in high power fields. Three independent pathologists evaluated the immunostaining results. For statistical analysis of CtBP2 stain, each slide was assessed using a semi-quantitative scoring system for both the intensity of the stain and the percentage of positive malignant cells. The intensity of staining was classified on a scale of 0 to 2 (0: negative or poor; 1: moderate; 2: strong). The percentage of tumor cells was scored as follows: 1 (0-49\%), 2 (50-74\%), and 3 (75-100\%). The total score was determined by multiplying these two scores, and samples were divided into 2 groups: high expression $(>3)$ and low expression $(\leq 3)$. When evaluating the Ki-67 stain, staining was scored in a semi-quantitative fashion. A cut-off value of $50 \%$ or more positively stained nuclei in five high-power fields was used to define Ki-67 staining; high expression group (50\%) and low expression group $(<50 \%)$.

Transient transfection. The CtBP2-shRNA and controlshRNA were purchased from Genechem (Shanghai, China). The CtBP2-specific shRNA target sequence was: CtBP2shRNA\#1 was 5'-CTTTGGATTCAGCGTCATA-3', CtBP2shRNA\#2 was 5'-TGAGAGTGATCGTGCGGAT-3', CtBP2shRNA\#3 was 5'-GACAGAATTTGTGAAGGTA-3', and CtBP2-shRNA\#4 was 5'-CTGCAATCTCAACGAACAT-3'.

When the cells reached $65 \%$ confluence, they were transfected with the CtBP2-shRNA or the control shRNA using Lipofectamine 2000 transfection (Invitrogen, Carlsbad, CA, USA) according to the manufacturer's protocol. Cells were harvested for western blot, CCK-8, flow cytometry, EdU and plate colony formation assays after transfection for $48 \mathrm{~h}$.

Cell cycle analysis. Starvation-refeeding was used for cell cycle analysis. The A549 cells were incubated without fetal bovine serum for $72 \mathrm{~h}$ to synchronize cells, then changed into complete medium and collected after $0,6,12,24$, and $48 \mathrm{~h}$. The cells were then fixed in $70 \%$ ethanol for at least $24 \mathrm{~h}$ at $-20^{\circ} \mathrm{C}$. Subsequently, the cells incubated with $1 \mathrm{mg} / \mathrm{ml} \mathrm{RNase} \mathrm{A}$ for $30 \mathrm{~min}$ at $37^{\circ} \mathrm{C}$ in PBS were stained with propidium iodide (PI, $50 \mu \mathrm{g} / \mathrm{ml}$ ) in PBS-Triton $\times 100$ for an additional $20 \mathrm{~min}$ at $4^{\circ} \mathrm{C}$, and analysed by BD FACScan (Becton-Dickinson, Franklin Lakes, NJ, USA). 
Cell counting kit-8 assays (CCK-8) and 5'-ethynyl2'-deoxyuridine (EdU) assay. A549 cells $\left(2 \times 10^{4} / 100 \mu \mathrm{l}\right)$ transfected with shRNA were seeded in 96-well plates and grown overnight. The CCK- 8 reagents were then added to a subset of the wells, followed by incubation in the dark at $37^{\circ} \mathrm{C}$ for $2 \mathrm{~h}$. Absorbency was measured at $450 \mathrm{~nm}$ using a microplate reader (Bio-Rad). The assay was performed in triplicate, values were recorded each day and growth curves were plotted. Cells proliferation was also tested by the EdU incorporation assay. Briefly, A549 cells $\left(4 \times 10^{4}\right.$ per well) were cultured in triplicate in 96-well plates and transfected with control shRNA or CtBP2-shRNA for 48h. Then, cells were incubated with $50 \mathrm{nmol}$ of EdU for an additional $2 \mathrm{~h}$ at $37^{\circ} \mathrm{C}$. The cells were fixed with $4 \%$ formaldehyde for $15 \mathrm{~min}$ at room temperature and treated with $0.5 \%$ Triton $\times 100$ for 20 min at room temperature to permeabilize cells. After being washed

Table 1. Expression of CtBP2 in 150 human NSCLC tissues.

\begin{tabular}{|c|c|c|c|c|}
\hline \multirow{2}{*}{ Characteristics } & \multicolumn{3}{|c|}{ CtBP2 expression } & \multirow{2}{*}{ p-value } \\
\hline & total & low & high & \\
\hline \multicolumn{5}{|l|}{ Age } \\
\hline$<60$ & 47 & 15 & 32 & 0.057 \\
\hline$\geq 60$ & 103 & 50 & 53 & \\
\hline \multicolumn{5}{|l|}{ Sex } \\
\hline Male & 121 & 55 & 66 & 0.284 \\
\hline Female & 29 & 10 & 19 & \\
\hline \multicolumn{5}{|l|}{ Histotype } \\
\hline Adenocarcinoma & 54 & 20 & 34 & 0.243 \\
\hline Squamous cell carcinoma & 96 & 45 & 51 & \\
\hline \multicolumn{5}{|l|}{ Smoking status } \\
\hline No & 97 & 44 & 53 & 0.498 \\
\hline Yes & 53 & 21 & 32 & \\
\hline \multicolumn{5}{|l|}{ Clinical stage } \\
\hline I & 65 & 43 & 22 & $<0.001^{*}$ \\
\hline II & 50 & 17 & 33 & \\
\hline III & 32 & 5 & 27 & \\
\hline IV & 3 & 0 & 3 & \\
\hline \multicolumn{5}{|l|}{ Histological differentiation } \\
\hline Well & 42 & 13 & 29 & $0.027^{*}$ \\
\hline Moderate & 94 & 42 & 52 & \\
\hline Poor & 14 & 10 & 4 & \\
\hline \multicolumn{5}{|l|}{ Tumor size $(\mathrm{cm})$} \\
\hline$\leq 3$ & 51 & 29 & 22 & $0.016^{*}$ \\
\hline$>3$ & 99 & 36 & 63 & \\
\hline \multicolumn{5}{|l|}{ Lymph node status } \\
\hline No & 92 & 51 & 41 & $<0.001^{*}$ \\
\hline Yes & 58 & 14 & 44 & \\
\hline \multicolumn{5}{|l|}{ ki67 } \\
\hline low expression & 71 & 42 & 29 & $<0.001^{\star}$ \\
\hline high expression & 79 & 23 & 56 & \\
\hline
\end{tabular}

* Statistical analyses were performed by the Pearson $\chi^{2}$ test and $p<0.05$ was considered significant with PBS three times, cells were incubated with $1 \times$ Apollo reaction cocktail $(100 \mu \mathrm{l} /$ well $)$ for $30 \mathrm{~min}$. DNA was stained with $10 \mu \mathrm{g} / \mathrm{ml}$ Hoechst 33342 stain $(100 \mu \mathrm{l} /$ well) for $20 \mathrm{~min}$ and visualized by fluorescence microscopy.

Colony formation assays. Cells were cultivated in 6-well culture plates at a density of 1000 cells/well after transfecting CtBP2-shRNA and control shRNA. After 2 weeks, cell colonies ( $\geq 50$ cells/colony) were counted by staining with a $0.5 \%$ crystal violet solution.

Wound healing assay. The migration ability of cells was measured using in vitro wound-healing assay. Cells were seeded into six-well plates and prepared until $80 \%$ growth confluence in a monolayer. Cells were serum starved for $12 \mathrm{~h}$ after $36 \mathrm{~h}$ transfection with control-shRNA, CtBP2-shRNA. Wounds were afflicted by scraping the monolayer cells with a sterile $100 \mu \mathrm{l}$ pipette tip. At 0,12 and $24 \mathrm{~h}$ after wounding, cells were observed under the inverted Leica phase-contrast microscope (Leica DFC $300 \mathrm{FX}$ ) to measure the distance between the two wounds at each time point and present the average percentage wound closure compared to that noted at zero time.

Statistical analysis. Statistical analysis was conducted by SPSS 22.0 statistical program. The expression of CtBP2 and clinical-pathological features was analyzed by 2 tests. Survival curves were obtained by the Kaplan-Meier method and analyzed by log-rank test. Multivariate analysis was performed using Cox's proportional hazards model. Values were expressed as mean \pm SEM and $p<0.05$ was considered statistically significant.

\section{Results}

High CtBP2 expression in human NSCLC tissues. To investigate whether the level of CtBP2 expression is associated with the progression of NSCLC, we first investigated the expression of CtBP2 on eight paired NSCLC tissues and the adjacent non-cancerous tissues, and the three A549, H1299 and SPCA-1 NSCLC cell lines were examined by western blotting. As shown in Figure 1A and 1B, CtBP2 was significantly over-expressed in NSCLC tissues compared to the adjacent non-tumor tissues. We also found that CtBP2 protein levels were up-regulated in NSCLC cell lines (Figure 1C). Moreover, 150 NSCLC samples were selected for immunohistochemical assay to examine the CtBP2, DvL1 and Ki-67 expression in clinical-pathological specimens. We found CtBP2 and Ki-67 immunoreactivity predominantly in the nucleus, and DvL1 in the Wnt/ $\beta$-catenin pathway was mainly located in the cytoplasm (Figure 1D). As expected, CtBP2 was up-regulated in poorly differentiated specimens compared to well-differentiated specimens, and this was consistent with the DvL1 and Ki-67 expression pattern (Figure 1D). Moreover, we found positive relationship between CtBP2 expression and Ki-67 using Spearman's rank correlation test (Figure 1E). These combined findings confirmed that CtBP2 is up-regulated in NSCLC tissues. 
A

CtBP2

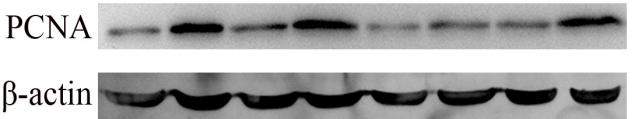

CtBP2 $=$

B

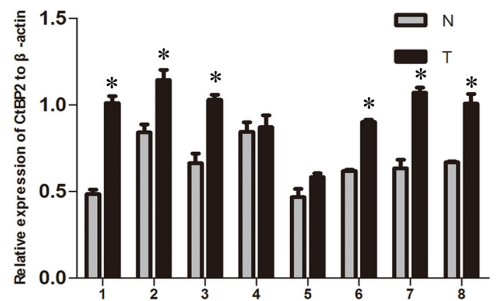

D

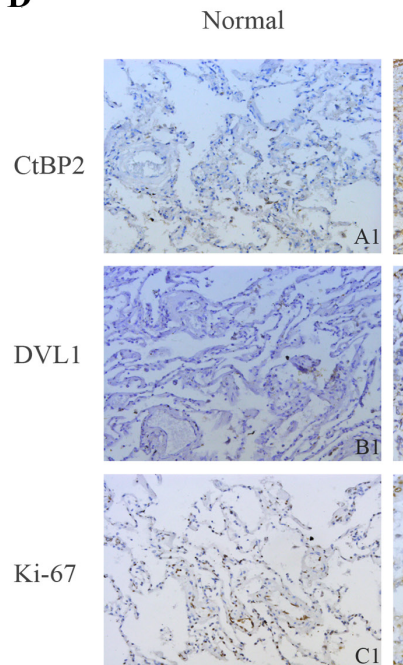

Well differentiated

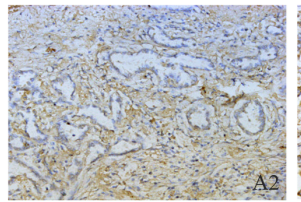

Mod differentiated

Poor differentiated
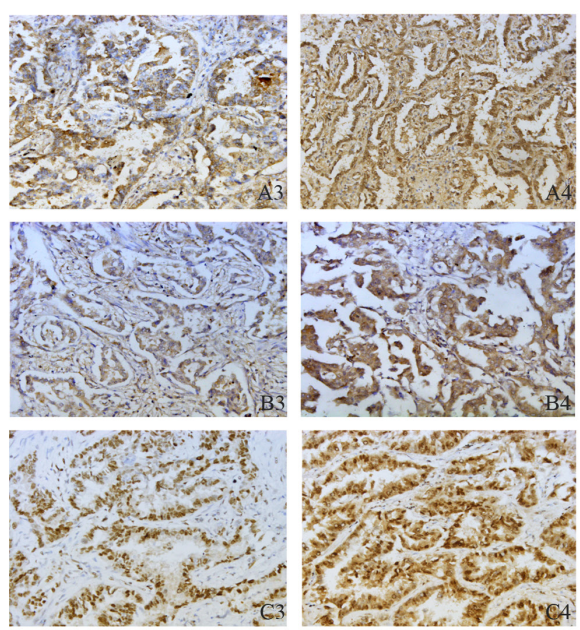

$\mathbf{E}$

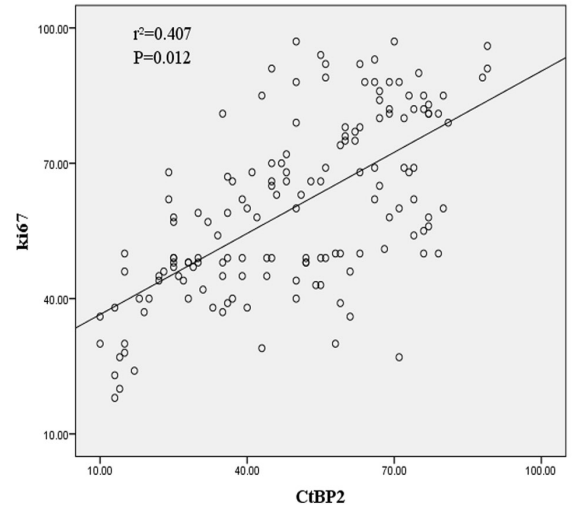

$c$

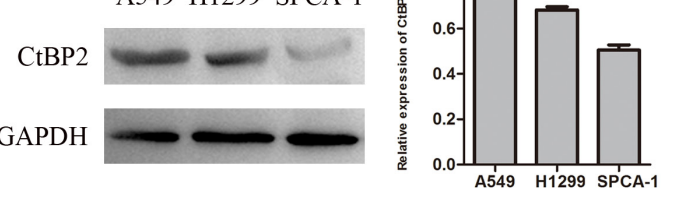

F

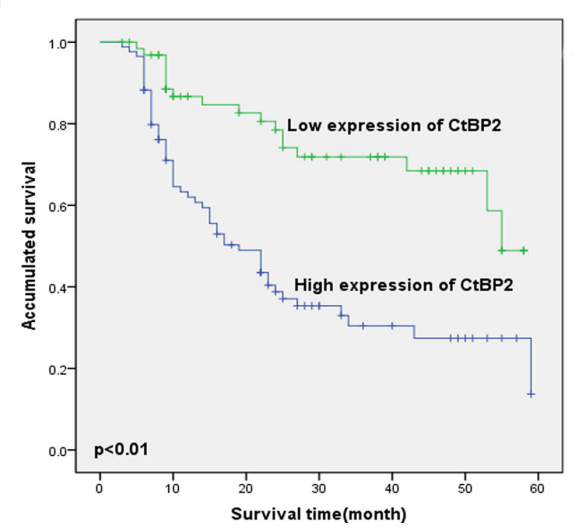

Figure 1. CtBP2 was over-expressed in NSCLC tissues and cells. A-C) Expression of CtBP2 in eight representative paired samples of NSCLC tissues (T), adjacent non-tumorous (N) tissues and three NSCLC cell. (B-D) The bar chart shows the ratio of CtBP2 protein to GAPDH by densitometry in the indicated cell lines. The data is mean \pm SEM. ${ }^{\star} \mathbf{P}<0.05$. D) Immunohistochemical stain of CtBP2, DvL1 and Ki-67 in adjacent normal tissues (A1, B1, C1), well differentiated (A2, B2, C2), moderate differentiated (A3, B3, C3) and poorly differentiated (A4, B4, C4) NSCLC tissues (Magnification bar = $100 \mu \mathrm{m}$ for all images). Scale bar $=100 \mu \mathrm{m}$ for all images. E) The correlation between CtBP2 and Ki-67 in NSCLC was further evaluated by Spearman's rank correlation test $(p=0.012)$. F) Kaplan-Meier survival curves for low CtBP2 expression versus high CtBP2 expression in 150 patients of NSCLC showed a highly significant separation $(\mathrm{p}<0.01)$. 

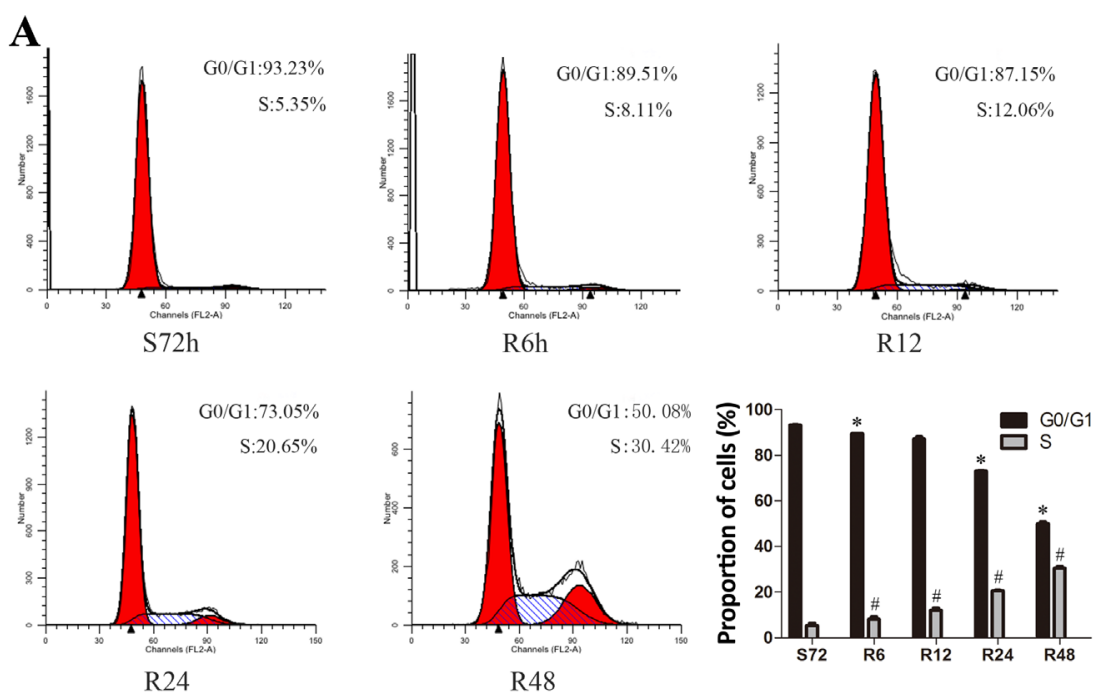

B
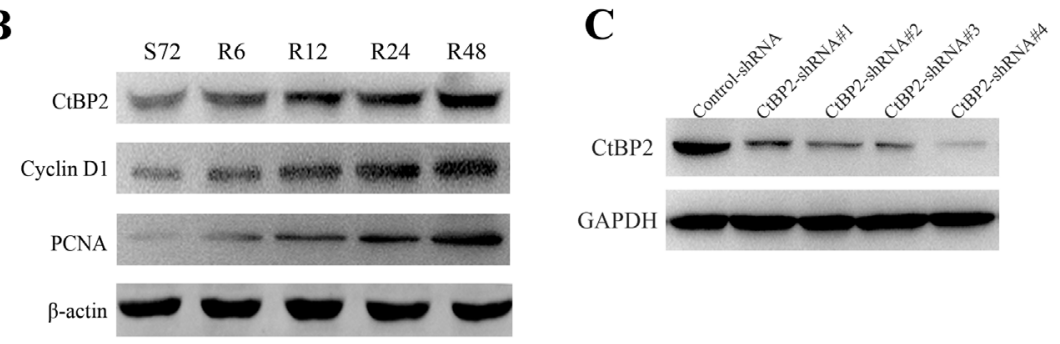

D

$\mathbf{E}$
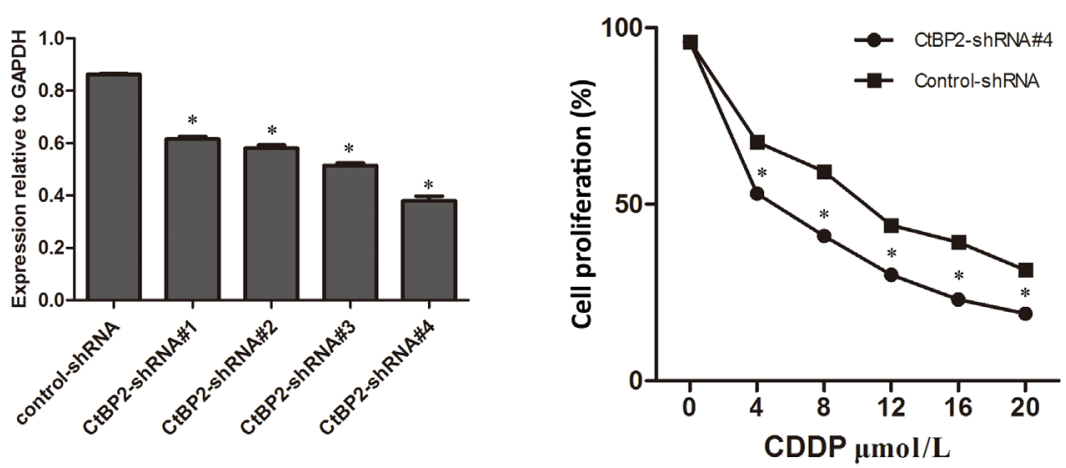

Figure 2. The expression of CtBP2 promotes proliferation and reduces sensitivity to chemotherapy drugs in A549 cells. A) Cells were synchronized at G0/G1 and progressed into the cell cycle when serum was added for S72h, R6h, R12h, R24h, and R48h. The experiment was conducted by flow cytometry. B) Western blot tested expression of CtBP2, cyclinD1, PCNA after 72 hours serum starvation in A549. S, serum starvation; R, serum release. C and D) CtBP2 expression was detected by western blot after transfection for 48 hours with CtBP2-shRNA in A549, while CtBP2-shRNA\#4 achieved the best-effected down-regulation. The relative level of CtBP2 was tested by densitometry. E) CCK-8 assays showing the A549 proliferation following addition of CDDP for the indicated concentration. 48 hours after CtBP2-shRNA\#4 transfection, cells were treated with different CDDP density stimulation. Then, CCK-8 assays compared the cell vitality of the CtBP2-shRNA\#4-transfected group with that of the control-shRNA group. Values are mean \pm SEM of three independent experiments; ${ }^{*}, * 00.05$.

CtBP2 expression correlates with NSCLC clinicalpathological features and survival. We evaluated the clinical-pathological significance of CtBP2 expression and physiological/pathological connection between CtBP2 and Ki-67 in NSCLC. Table 1 highlights that CtBP2 expression is significantly associated with lymph node metastasis, tumor size, clinical stage, Ki-67 expression and histological differentiation $(\mathrm{p}<0.05)$. Kaplan-Meier analysis computed the association between the CtBP2 expression and patient survival (Figure 1F). The survival curves showed that high CtBP2 expression significantly decreased overall survival compared to low expression. Pearson $\chi 2$ tests are shown in Table 2, 
and univariate survival analysis showed that clinical stage, tumor size, lymph node metastasis, histological differentiation, Ki-67 expression and CtBP2 expression were prognostic factors for overall survival $(\mathrm{p}<0.05)$. Moreover, the Cox proportional hazards model revealed that CtBP2 expression and histological differentiation were independent prognostic factors for overall NSCLC patient survival $(\mathrm{p}<0.05)$ (Table 3 ). The over-expression of CtBP2 could be a strong indicator of poor NSCLC prognosis.

CtBP2 expression promotes proliferation and reduces CDDP sensitivity in NSCLC cells. Since CtBP2 expression positively correlated with Ki-67 and PCNA expression
(Figures $1 \mathrm{~A}$ and 1D), and these are both cell proliferation markers, we predicted that CtBP2 has a significant role in NSCLC cell-cycle progression. To verify the role of CtBP2 in cycle progression, we chose A549 for the serum starvation and re-feeding process and these cells were arrested in the G1 phase after 72 hours serum starvation. Upon serum addition, A549 cells were released from the G0/G1 phase and gradually entered the $S$ phase (Figure 2A). To further confirm this hypothesis, western blot analyzed PCNA and CtBP2 expression and determined that the expression of PCNA and cell cycle regulator Cyclin D1 were up-regulated after serum stimulation in A549 cells, and concomitant up-regulation of

$\mathbf{A}$

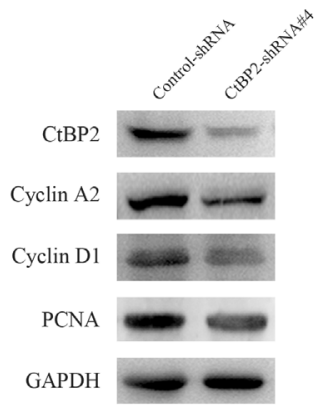

B

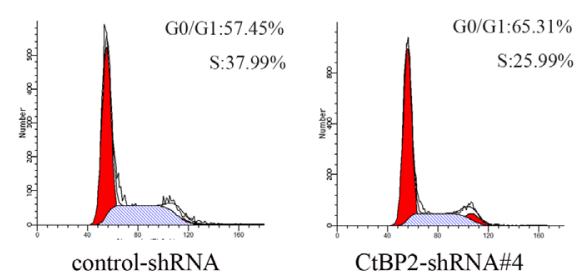

C

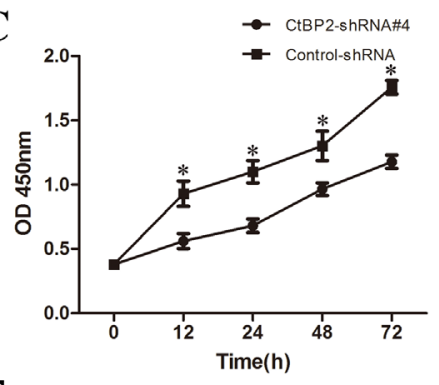

$\mathbf{E}$

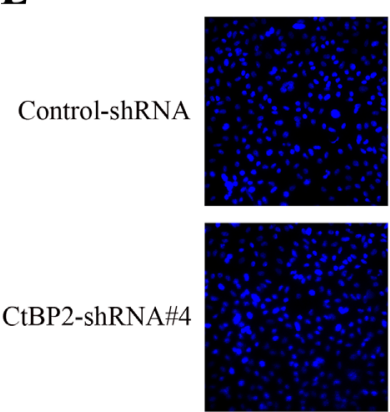

Hoechst 33342
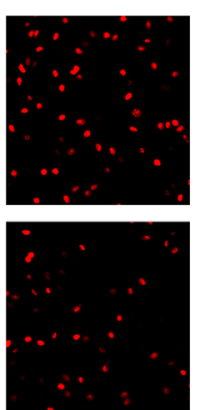

EdU
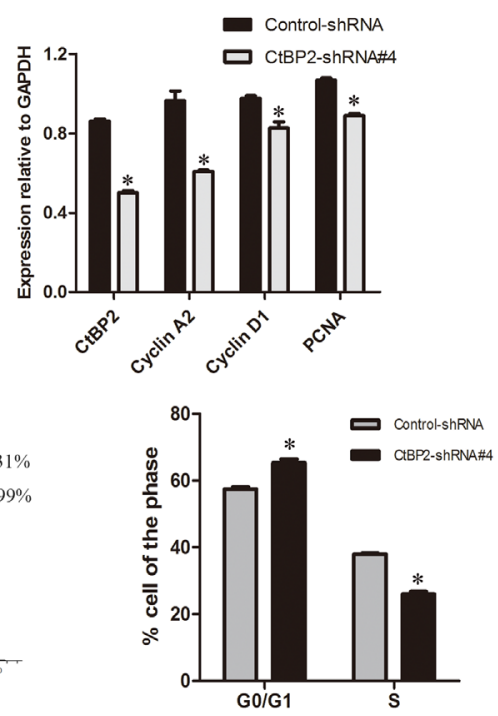

D
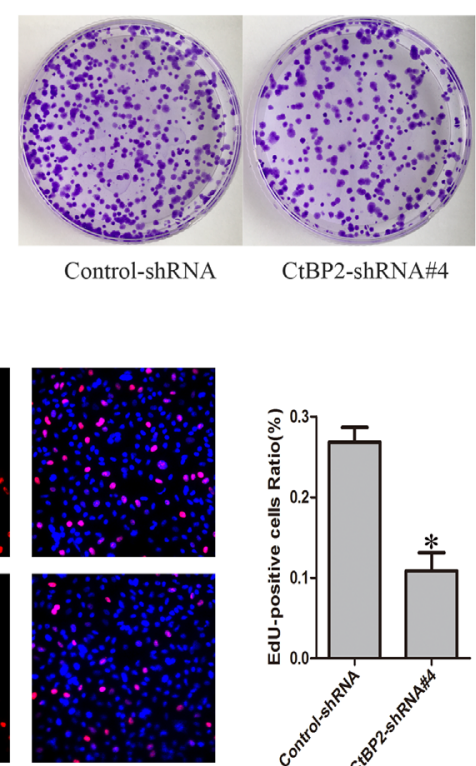

Merged

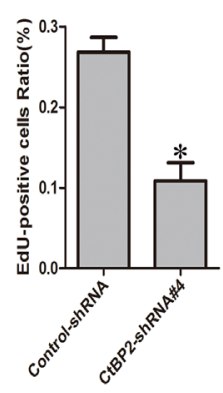

Figure 3. Knockdown of CtBP2 inhibits A549 cells proliferation. A) Western blots showed that the expression of cell cycle-related proteins such as cyclinA2, cyclinD1 and PCNA declined in CtBP2shRNA\#4-treated A549 cells. The relative expression levels were shown by density photometry. B) Cell cycle analysis was performed after knockdown of CtBP2 in A549, and the ratio of colorimetric density of the cell cycle. C-D) CCK-8 assay and colony formation assay showed that A549 treated with CtBP2-shRNA\#4 revealed remarkably weakened proliferation. Absorbance was used to examine the control-shRNA and CtBP2-shRNA\#4-treated A549. E) Fewer EdUlabelled cells fluoresced red in the CtBP2-shRNA\#4 treatment; indicating that proliferation is inhibited following transfection of CtBP2-shRNA for 48 hours. All cell nuclei had blue flourescence from Hoechst 33342 staining (original magnification $\times 20$ ). The bar chart presents the ratio of EdU-labeled cells to Hoechst 33342-labelled cells. The data presented is mean \pm SEM. ${ }^{\star} \mathbf{P}<0.05$ compared with the control. The same experiment was repeated at least three times. 


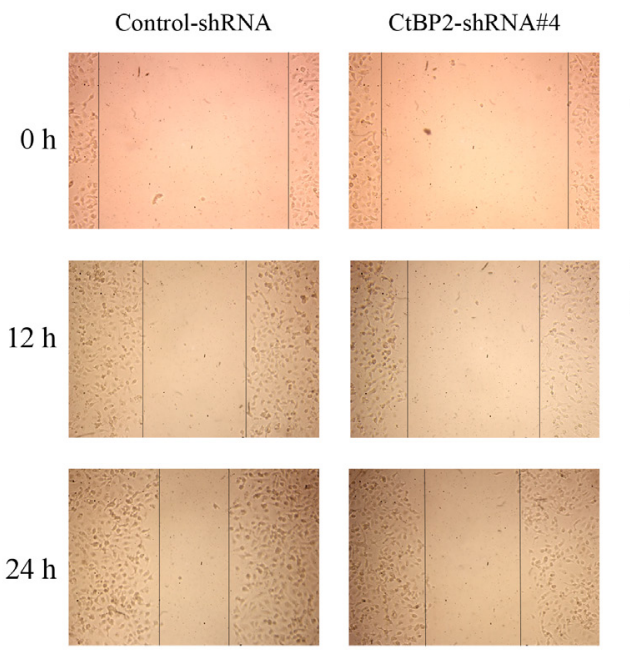

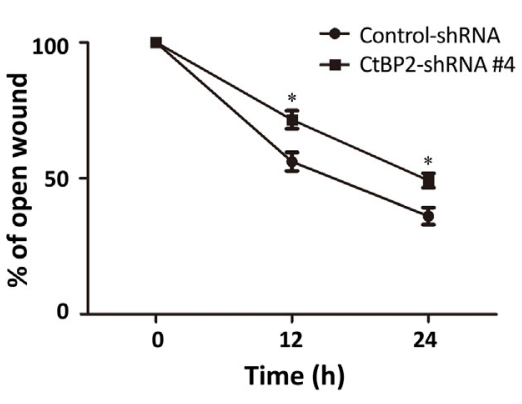

Figure 4. Knockdown of CtBP2 inhibits A549 cells migration. A549 cells expressing Control or CtBP2-shRNA\#4 were scratched by a $100-\mu \mathrm{L}$ pipette tip and cultured for 24 hours. Representative images of wound healing were shown. The relative ratio of wound closure per field was shown at $0,12,24$ hours. The data is mean $\pm S E M .{ }^{*} p<0.05$. The same experiment was repeated at least three times.
CtBP2 was also observed (Figure 2B). This result indicates that $\mathrm{CtBP} 2$ could have a role as a positive regulator of NSCLC cells in a cell cycle-dependent pathway.

We then transiently transfected A549 cells with CtBP2shRNA\#1， CtBP2-shRNA\#2， CtBP2-shRNA\#3， CtBP2shRNA\#4 and the control-shRNA to further investigate the role of CtBP2 in cellular proliferation. After 48 hours, Western blot evaluated the efficiency of transfection. We found that CtBP2 protein levels decreased most in A549 infected with CtBP2-shRNA\#4 compared to cells treated with sh-CtBP2 (Figures 2C and 2D). We then used CtBP2-shRNA\#4 and established that Cis-diamminedichloroplatinum (CDDP) has an important role in cancer chemotherapy and activity in a wide variety of tu mors [19]. We used cell vitality assays in A549 cells treated with different concentrations of CDDP to research NSCLC cells for sensitivity to CDDP. We identified that the cell-proliferation rate was reduced in a dosedependent manner, and drug sensitivity achieved maximum at $20 \mu \mathrm{mol} / \mathrm{l}$.

To further confirm the contribution of CtBP2 to CDDP sensitivity in NSCLC cells, we measured the proliferation of NSCLC cells by CtBP2 knock-down and treatment with CDDP for 24 hours. Proliferation reached its lowest level at $20 \mu \mathrm{mol} / \mathrm{l}$ concentration, and it was further enhanced following CtBP2 knockdown (Figure 2E). These combined results indicate that $\mathrm{CtBP} 2$ expression can reduce NSCLC cells sensitivity to CDDP.

CtBP2 knockdown inhibits NSCLC cell proliferation and migration. We investigated the role of CtBP2 on cell proliferation in NSCLC by transfecting A549 cells with control-shRNA and CtBP2-shRNA\#4. Figure 3A highlights that expression of cyclinA2, cyclinD1 and PCNA in cells transfected with CtBP2-shRNA\#4 was significantly decreased. Flow cytometric cell cycle analysis then revealed an increase in the number of cells in the G0/G1 phase from $57.45 \%$ to $65.31 \%$ and a decrease in cell number in the $S$ phase from $37.99 \%$ to $25.99 \%$ (Figure 3B). This indicates that down-regulation of CtBP2 slows the NSCLC cell cycle. Moreover, CCK- 8 assay confirmed the effect of CtBP2. Here, $\mathrm{CtBP} 2$ knockdown reduced cell proliferation (Figure 3C). Colony formation assay then showed that the colony formation rate was markedly attenuated in CtBP2 knockdown (Figure 3D), and EdU assay also revealed significant decrease in cell proliferation after CtBP2 knockdown (Figure 3E).

It is well known that tumor cell migration is a key factor in their invasive phenotype. We performed wound healing assays to determine the influence of CtBP2 in promoting cell migration, and found that A549 cell lines transfected with CtBP2-shRNA\#4 had drastically minor migration ability compared to controls at 12 and 24 hours after scratching (Figure 4). These combined results suggest that CtBP2 knockdown delays G0/G1-S transition and also inhibits NSCLC cell proliferation and migration.

CtBP2 interacts with DvL1 to activate the Wnt/ $\beta$ catenin signaling pathway, leading to enhanced cell vitality and reduced CDDP sensitivity in NSCLC cells. Numerous studies have reported that the Wnt/ $\beta$-catenin signaling pathway is abnormally active in NSCLC, and that this is closely related to NSCLC proliferation and growth. DvL1 is also a key molecule in the classical Wnt/ $\beta$-catenin signaling pathway, and some studies suggest that DvL1 is closely related to proliferation and growth in NSCLC. We therefore performed immunoprecipitation to further probe the mechanism of CtBP2 on cellular proliferation and drug sensitivity. As shown in Figure 5A, CtBP2 can co-immunoprecipitate with the anti-DvL1 antibody and encourage the naturally occurring interaction between endogenous CtBP2 and DvL1 in vivo; and vice versa. 
To evaluate whether loss of DvL1 activation was involved in the effects induced by CtBP2 knockdown, we investigated DvL1 and the expression of its target genes. We observed that knockdown of CtBP2 inhibited the activation of DvL1 and decreased the expression of $\beta$-catenin, $\mathrm{C}$-myc and Cyclin D1 in A549cells and that both of these were $\beta$-catenin/TCF target genes (Figure 5B and 5C). This result showed CtBP2 enhanced cell vitality through the $\mathrm{Wnt} / \beta$-catenin pathway.

To test if CtBP2-induced reduced CDDP sensitivity is dependent on the Wnt/ $\beta$-catenin pathway, A549 cells were treated with CDDP for 24 hours. Western blot examined the expression of DvL1, $\beta$-catenin and the cleaved-PARP apoptosis marker. As shown in Figure 5D, CtBP2-shRNA\#4 combined with CDDP treatment decreased the levels of DvL1 and $\beta$-catenin, but up-regulated the cleaved-PARP. We also found that after CDDP treatment, the cell vitality in CtBP2-silenced cells was reduced after CDDP treatment compared to controls because of reduced Wnt $/ \beta$-catenin pathway activation (Figure 5E). These combined results show that CtBP2 enhances cell vitality and reduces CDDP sensitivity by activating the $\mathrm{Wnt} / \beta$-catenin pathway.

\section{Discussion}

The occurrence and progression of NSCLC is a complex, multi-factorial process. Although diagnosis and treatment have made great progress, the overall survival rate of patients is low because of the high rate of tumor relapse, invasion and metastasis. The occurrence of NSCLC is closely linked to the cell cycle, and abnormal proliferation is the most significant difference between NSCLC and normal cells. Therefore, inhibition of NSCLC cell proliferation is an important aspect of anti-tumor therapy. The reduced drug sensitivity of chemotherapy also brings problems, and therefore it is imperative to determine novel therapeutic targets and exploit new anti-cancer treatments. In our study, we verified that CtBP2 could be a significant regulator in NSCLC cell proliferation and drug sensitivity.

Previous studies showed that CtBP2 is closely related to human cancers and that it is inversely associated with expression of the p16INK4 tumor suppressor gene. CtBP2 can inhibit the up-regulation of p16INK4A induced by cisplatin, thereby promoting the proliferation of esophageal cancer cells [20]. In ovarian cancer, Barroilhet found that CtBP2 reduced sensitivity of ovarian cancer cells to anti-neoplastic drugs [21]. While the precise roles of CtBP2 in NSCLC remain unknown, we found that $\mathrm{CtBP} 2$ was over-expressed in NSCLC tumor tissues and cell lines and that it is related to NSCLC clinical pathologic variables.

Multivariate analysis indicated that CtBP2 could be an independent prognostic factor for NSCLC patient survival, and the CtBP2-silencing experiment resulted in reduced cell proliferation and enhanced CDDP sensitivity. Therefore, CtBP2 may be engaged in the evolution of NSCLC tumorigenesis.
Table 2. Contribution of various potential prognostic factors to survival by univariate analysis in 150 NSCLC specimens.

\begin{tabular}{|c|c|c|c|c|}
\hline \multirow{2}{*}{ Characteristics } & \multicolumn{3}{|c|}{ Survival status } & \multirow{2}{*}{ p-value } \\
\hline & total & Alive & Dead & \\
\hline \multicolumn{5}{|l|}{ Age } \\
\hline$<60$ & 47 & 20 & 27 & 0.118 \\
\hline$\geq 60$ & 103 & 58 & 45 & \\
\hline \multicolumn{5}{|l|}{ Sex } \\
\hline Male & 121 & 63 & 58 & 0.974 \\
\hline Female & 29 & 15 & 14 & \\
\hline \multicolumn{5}{|l|}{ Histotype } \\
\hline Adenocarcinoma & 54 & 27 & 27 & 0.713 \\
\hline Squamous cell carcinoma & 96 & 51 & 45 & \\
\hline \multicolumn{5}{|l|}{ Smoking status } \\
\hline No & 97 & 52 & 45 & 0.594 \\
\hline Yes & 53 & 26 & 27 & \\
\hline \multicolumn{5}{|l|}{ Clinical stage } \\
\hline I & 65 & 45 & 20 & $0.001^{\star}$ \\
\hline II & 50 & 23 & 27 & \\
\hline III & 32 & 10 & 22 & \\
\hline IV & 3 & 0 & 3 & \\
\hline \multicolumn{5}{|l|}{ Histological differentiation } \\
\hline Well & 10 & 2 & 4 & $<0.001^{\star}$ \\
\hline Moderate & 94 & 66 & 28 & \\
\hline Poor & 42 & 2 & 40 & \\
\hline \multicolumn{5}{|l|}{ Tumor size $(\mathrm{cm})$} \\
\hline$\leq 3$ & 51 & 33 & 18 & $0.025^{\star}$ \\
\hline$>3$ & 99 & 45 & 54 & \\
\hline \multicolumn{5}{|l|}{ Lymph node status } \\
\hline No & 92 & 57 & 35 & $0.002^{*}$ \\
\hline Yes & 58 & 21 & 37 & \\
\hline \multicolumn{5}{|l|}{$\mathrm{CtBP} 2$} \\
\hline low expression & 65 & 47 & 18 & $<0.001^{\star}$ \\
\hline high expression & 85 & 31 & 54 & \\
\hline \multicolumn{5}{|l|}{ ki67 } \\
\hline low expression & 71 & 43 & 28 & $0.047^{\star}$ \\
\hline high expression & 79 & 35 & 44 & \\
\hline
\end{tabular}

* Statistical analyses were performed by the Pearson $\chi^{2}$ test and $\mathrm{p}<0.05$ was considered significant

Table 3. Contribution of various potential prognostic factors to survival by Cox regression analysis in 150 NSCLC specimens.

\begin{tabular}{lccc}
\hline Characteristics & $\begin{array}{c}\text { Hazard } \\
\text { Ratio }\end{array}$ & $\begin{array}{c}\mathbf{9 5 . 0 \%} \\
\text { Confidence Interval }\end{array}$ & p-value \\
\hline Clinical stage & 0.461 & $0.116-1.828$ & 0.270 \\
Histological & 4.332 & $1.076-11.561$ & $0.037^{*}$ \\
differentiation & & & \\
Lymph node status & 0.559 & $0.322-1.112$ & 0.105 \\
Tumor size (cm) & 1.038 & $0.58-1.859$ & 0.900 \\
CtBP2 & 2.483 & $1.274-4.838$ & $0.008^{*}$ \\
Ki-67 & 1.300 & $0.757-2.231$ & 0.342 \\
\hline
\end{tabular}

${ }^{*}$ Statistical analyses were performed by the Cox regression analysis and $\mathrm{p}<0.05$ was considered significant 
A

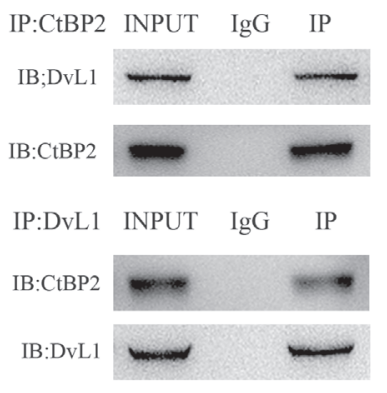

C

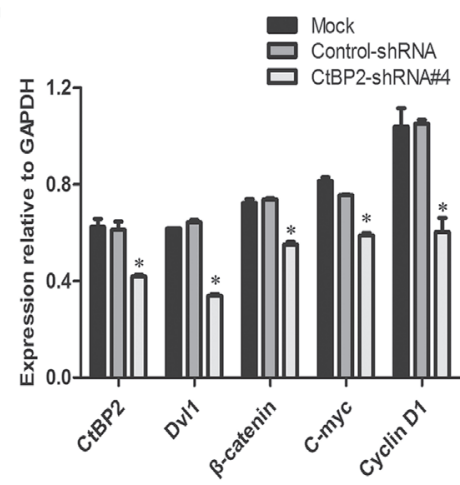

$\mathbf{E}$

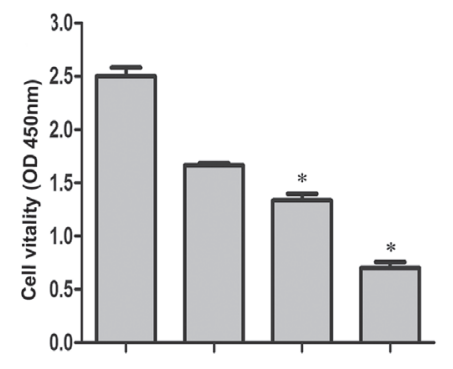

B

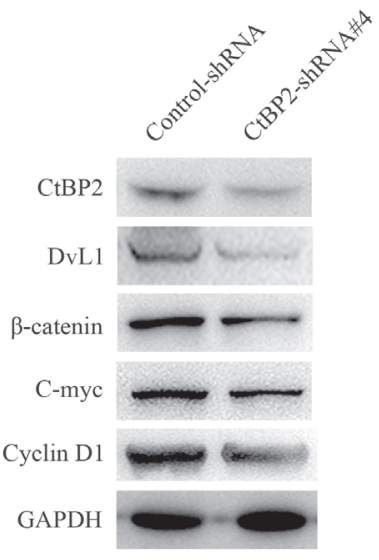

D

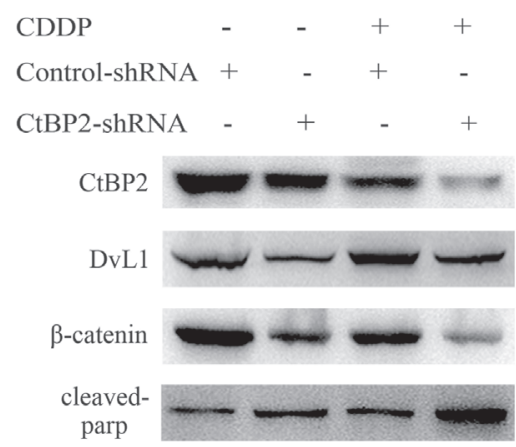

GAPDH

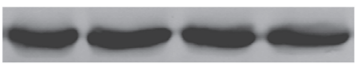

Control-shRNA

CtBP2-shRNA

Figure 5. Interaction of CtBP2 with DvL1 mediated activation of the Wnt/ $\beta$-catenin pathway enhanced cell vitality and reduced CDDP sensitivity in NSCLC. A) Co-immunoprecipitation assay was used for the analysis of interaction between CtBP2 and DvL1. B) Western blot showed that the expression of DvL1, $\beta$-catenin and C-myc, the members of the Wnt/ $\beta$-catenin family, and the expression of cell cycle-related proteins such as PCNA declined in CtBP2-shRNA\#4-treated A549 cells. C) The ratio of colorimetric density of CtBP2 and downstream genes. D) Western blot analysis of DvL1, $\beta$-catenin, and cleaved PARP expression of A549 when CtBP2 is down-regulated and with or without CDDP treatment. E) CCK-8 assays were performed to compare the cell vitality of the CtBP2-shRNA\#4-transfected group with that of the control-shRNA group, and then the samples were subjected to treatment with or without CDDP for 24 hours, respectively. The data is in mean \pm SEM. ${ }^{\star}$ p $<0.05$ compared to controls.

Numerous studies have revealed that canonical Wnt $/ \beta$ catenin signaling is a classic pathway with crucial roles in NSCLC progression. DvL1 and DvL2 are regulators or downstream molecules of $\mathrm{Wnt} / \beta$-catenin signaling, and previous studies have shown that DvL interacts with c-Jun and $\beta$-catenin in the nucleus, forming a stable DvL/C-Jun$\mathrm{P} / \mathrm{Tcf} / \beta$-catenin complex that activates transcription of the
Wnt target gene [22]. In addition, FOXKs family transcription factor interacted with DvL2 in the nucleus to promote the Wnt/ $\beta$-catenin signaling pathway [23]. DvL2 has similar sequence to DvL1 and this suggests they are functionally related genes. We further demonstrated that CtBP2 interacts with DvL1 in NSCLC cells, and CtBP2 knockdown can inhibit DvL1 activity and suppress the expres- 
sion of downstream targets such as $\beta$-catenin. While this indicates that CtBP2 may affect cell vitality and drug sensitivity through the Wnt/ $\beta$-catenin signaling pathway, further studies are required to clarify the molecular mechanisms of CtBP2 in NSCLC pathogenesis.

In conclusion, our combined results confirmed that CtBP2 can up-regulate DvL1 expression and activate the Wnt $/ \beta$-catenin signaling pathway; ultimately promoting cell proliferation and reducing NSCLC cell sensitivity to CDDP. Therefore, CtBP2 could be an original and successful molecular target in the diagnosis and therapy of non-small cell lung cancer.

Acknowledgements: The present study was supported by the National Natural Science Foundation of China (No.81472185), National Natural Science Foundation of China (No.81572267).

\section{References}

[1] CHEN W, ZHENG R, ZENG H, ZHANG S. The updated incidences and mortalities of major cancers in China, 2011. Chin J Cancer 2015; 34: 502-507. https://doi.org/10.1186/ s40880-015-0042-6

[2] ETTINGER DS1, AKERLEY W, BEPLER G, BLUM MG, CHANG A et al. Non-small cell lung cancer. J Natl Compr Canc Netw 2010; 8: 740-801.

[3] JEMAL A, SIEGEL R, WARD E, MURRAY T, XU J et al. Cancer statistics, 2006. CA Cancer J Clin 2006; 56: 106-130.

[4] SHI Y, SAWADA J, SUI G, AFFAR EL B, WHETSTINE JR et al. Coordinated histone modifications mediated by a CtBP co-repressor complex. Nature 2003; 422: 735-738. https:// doi.org/10.1038/nature 01550

[5] ZHAO LJ, SUBRAMANIAN T, ZHOU Y, CHINNADURAI G. Acetylation by p300 regulates nuclear localization and function of the transcriptional corepressor CtBP2. J Biol Chem 2006; 281: 4183-4189. https://doi.org/10.1074/jbc. M509051200

[6] BERGMAN LM, BIRTS CN, DARLEY M, GABRIELLI B, BLAYDES JP et al. CtBPs promote cell survival through the maintenance of mitotic fidelity. Mol Cell Biol 2009; 29: 4539_ 4551. https://doi.org/10.1128/MCB.00439-09

[7] CHINNADURAI G. The transcriptional corepressor CtBP: a foe of multiple tumor suppressors. Cancer Res 2009; 69: 731-734. https://doi.org/10.1158/0008-5472.CAN-08-3349

[8] FANG M, LI J, BLAUWKAMP T, BHAMBHANI C, CAMPBELL $N$ et al. C-terminal-binding protein directly activates and represses Wnt transcriptional targets in Drosophila. EMBO J 2006; 25: 2735-2745. https://doi.org/10.1038/ sj.emboj.7601153

[9] DAI F, XUAN Y, JIN JJ, YU S, LONG ZW et al. CtBP2 overexpression promotes tumor cell proliferation and invasion in gastric cancer and is associated with poor prognosis. Oncotarget 2017; 8: 28736-28749. https://doi.org/10.18632/oncotarget.15661

[10] TAKAYAMA K, SUZUKI T, FUJIMURA T, URANO T, TAKAHASHI $S$ et al. CtBP2 modulates the androgen receptor to promote prostate cancer progression. Cancer Res 2014; 74: 6542-6553. https://doi.org/10.1158/0008-5472.CAN-14-1030
[11] ZHANG G, KANG L, CHEN J, XUE Y, YANG $M$ et al. CtBP2 Regulates TGFbeta2-Induced Epithelial-Mesenchymal Transition Through Notch Signaling Pathway in Lens Epithelial Cells. Curr Eye Res 2016; 41: 1057-1063. https:// doi.org/10.3109/02713683.2015.1092554

[12] ZHENG X, SONG T, DOU C, JIA Y, LIU Q. CtBP2 is an independent prognostic marker that promotes GLI1 induced epithelial-mesenchymal transition in hepatocellular carcinoma. Oncotarget 2015; 6: 3752-3769. https://doi. org/10.18632/oncotarget.2915

[13] ZHANG Y, KWOK JS, CHOI PW, LIU M, YANG J et al. Pinin interacts with C-terminal binding proteins for RNA alternative splicing and epithelial cell identity of human ovarian cancer cells. Oncotarget 2016; 7: 11397-11411. https:// doi.org/10.18632/oncotarget.7242

[14] PAI SG, CARNEIRO BA, MOTA JM, COSTA R, LEITE CA et al. Wnt/beta-catenin pathway: modulating anticancer immune response. J Hematol Oncol 2017; 10: 101. https://doi. org/10.1186/s13045-017-0471-6

[15] KAFKA A, BASIC-KINDA S, PECINA-SLAUS N. The cellular story of dishevelleds. Croatian Medical Journal 2014; 55: 459-467.

[16] KAFKA A, TOMAS D, BEROS V, PECINA HI, ZELJKO $\mathrm{M}$ et al. Brain metastases from lung cancer show increased expression of DVL1, DVL3 and beta-catenin and down-regulation of E-cadherin. Int J Mol Sci 2014; 15: 10635-10651. https://doi.org/10.3390/ijms150610635

[17] ZHAO Y, YANG ZQ, WANG Y, MIAO Y, LIU Y et al. Dishevelled-1 and dishevelled-3 affect cell invasion mainly through canonical and noncanonical Wnt pathway, respectively, and associate with poor prognosis in nonsmall cell lung cancer. Mol Carcinog 2010; 49: 760-770. https://doi. org/10.1002/mc.20651

[18] GU S, ZHANG R, GU J, LI X, LV L et al. HES5 promotes cellular proliferation of non-small cell lung cancer through STAT3 signaling. Oncol Rep 2017; 37: 474-482. https://doi. org/10.3892/or.2016.5268

[19] AHMAD S. Platinum-DNA interactions and subsequent cellular processes controlling sensitivity to anticancer platinum complexes. Chem Biodivers 2010; 7: 543-566. https://doi. org/10.1002/cbdv.200800340

[20] GUAN C, SHI H, WANG H, ZHANG J, NI W et al. CtBP2 contributes to malignant development of human esophageal squamous cell carcinoma by regulation of p16INK4A. J Cell Biochem 2013; 114: 1343-1354. https://doi.org/10.1002/ jcb. 24475

[21] BARROILHET L, YANG J, HASSELBLATT K, PARANAL RM, NG SK et al. C-terminal binding protein-2 regulates response of epithelial ovarian cancer cells to histone deacetylase inhibitors. Oncogene 2013; 32: 3896-3903. https://doi. org/10.1038/onc.2012.380

[22] GAN XQ, WANG JY, XI Y, WU ZL, LI YP et al. Nuclear Dvl, c-Jun, beta-catenin, and TCF form a complex leading to stabilization of beta-catenin-TCF interaction. J Cell Biol 2008; 180: 1087-1100. https://doi.org/10.1083/jcb.200710050

[23] WANG W, LI X, LEE M, JUN S, AZIZ KE et al. FOXKs promote Wnt/beta-catenin signaling by translocating DVL into the nucleus. Dev Cell 2015; 32: 707-718. https://doi. org/10.1016/j.devcel.2015.01.031 